\title{
Identidad y sentidos subjetivos sobre la prostitución en mujeres venezolanas que la ejercen
}

\author{
Identity and subjective senses about prostitution in Venezuelan \\ women who practice it
}

Freddy Rafael Suárez Álvarez

Recibido: 23/03/2019

Aceptado: $13 / 12 / 2020$

\section{RESUMEN}

La prostitución se caracteriza por la exclusión, marginación, prejuicios y estigmatización hacia estas personas. El objetivo de la investigación es conocer la identidad y el sentido subjetivo sobre la prostitución en las mujeres que lo ejercen. El diseño es cualitativo, paradigma interpretativo y método fenomenológico. Se realizó entrevistas a 4 mujeres que se dedican a la prostitución y se eligieron intencionalmente. Existe una ambivalencia en la identidad y el sentido subjetivo sobre la prostitución, ya que se encuentran atrapadas entre el dilema de la construcción propia sobre sí mismas y la construcción familiar, social, cultural e histórica con que se define el modelo de mujer y el mundo de la prostitución. La interrelación de todas estas estructuras viene a formar una identidad y sentido subjetivo de la prostitución confusa, ya que responden a un sistema de pensamiento patriarcal que va en discordancia con el ideal de mujer y de madre.

Palabras clave: subjetividad, identidad, prostitución, psicología, psicología social.

\begin{abstract}
Prostitution is characterized by exclusion, marginalization, prejudice and stigmatization towards these people. The objective of the research is to know the identity and subjective sense of prostitution in women who practice it. The design is qualitative, interpretive paradigm and phenomenological method. Interviews were conducted with 4 women engaged in prostitution and intentionally chosen. There is an ambivalence in the identity and the subjective sense of prostitution, since they are caught between the dilemma of selfconstruction on themselves and the family, social, cultural and historical construction with which the model of women and the world is defined of prostitution. The interrelation of all these structures comes to form an identity and subjective sense of confused prostitution, since they respond to a patriarchal system of thought that goes in discordance with the ideal of woman and mother.
\end{abstract}

Keywords: Subjectivity, Identity, Prostitution, Psychology, Social Psychology.

Freddy Rafael Suárez Álvarez es licenciado en Filosofía y Psicología y en profesor de Psicología en la Universidad Centroccidental Lisandro Alvarado (Venezuela). Correo electrónico: freddysuarez17@gmail.com. ID: https://orcid.org/0000-0001-7134-9881 


\section{INTRODUCCIÓN}

La prostitución es una realidad visible a nivel mundial que actualmente se oculta en diversas realidades bajo las denominaciones de masajistas, camareras, damas de compañía, entre otras. Se estima que genera al menos siete billones de dólares anuales a nivel mundial y se espera que vaya en aumento, esta cifra viene como consecuencia del gran número de consumidores que solicitan dichos servicios (Gómez y Verdugo, 2015).

Existe una gran diversidad de paradigmas que sostienen que la prostitución viene dada por el poco acceso de la mujer a un trabajo digno y de calidad; otros como una opción laboral; otros como la explotación de la mujer como objeto sexual, entre otros. En lo se tiene un acuerdo, es que actualmente esta realidad se vive en todos los países (Fernández, 2011).

Se podría decir que lo que determina este punto del mercado es el intercambio financiero, a través de una transacción económica por el cuerpo (Asociación Vivre Ensemble Education, 2014). Es por ello que algunos lo definen como entregar y explotar el cuerpo humano para ofrecer servicios sexuales, con fines de lucro (Fundation Scelles, 2012); o como el intercambio de bienes materiales o económicos, por la obtención de placer del prostituidor, a través de cualquier parte del cuerpo de otra persona (Díez, 2012).

Sin embargo, para efectos de esta investigación se definirá como el acceso grupal y regulado al cuerpo de las mujeres por parte de los hombres, alegando que es grupal en el sentido de que el cuerpo femenino pasa a ser un bien público y es por ello que cualquier hombre (o grupos de hombres) tiene acceso al mismo, y por otro lado, es regulado porque responde a una serie de normas ya preestablecidas. Teniendo en cuenta el factor económico, lo más importante es el libre acceso al cuerpo de la mujer (Álvarez, 2012).

Según Ulloa (2011), en América Latina es proveniente de la época de la colonización, en el cual la mujer era intercambiada como forma de remuneración para los impuestos de las guerras. Brasil es un país donde la prostitución va aumentando cada vez más, debido a que la ubicación geográfica del Centro-Oeste del país, funciona como surtidora del comercio sexual (de Matos. et al., 2013). Por otra parte en Colombia, esta actividad se relaciona con 
el grado de instrucción y la pobreza, lo que obliga a mantener el ejercicio de esta práctica debido a su desventaja socioeconómica y cultural (Condiza y Hernández, 2012).

En Venezuela, al igual que en los demás países del mundo, quienes solicitan los servicios de prostitución principalmente son los hombres, y las personas que la practican son en su mayoría mujeres (Observatorio Venezolano de los Derechos Humanos de las Mujeres [OVDHM], 2014).

Es importante resaltar que en Venezuela es notable la gran cantidad de mujeres que se dedican a la prostitución por diversos factores, pero principalmente lo que ha llevado a la población femenina a ingresar a este mundo son el sostenimiento de sus hijos, de sus familiares y las dificultades económicas que se vive dentro del hogar (Dakduk, 2007), aunado a esto la crisis que vive el país es otro factor importante para la inclusión de las personas en este ambiente.

Tampoco se conoce en Venezuela "el número de éstas, cantidad de clientes que atienden, condiciones de trabajo, prácticas sexuales o servicios que prestan, prevalencia e incidencia del VIH entre las mismas" (Aragón, 2000, p. 59), sin contar los escasos estudios sobre este tema en el país (Mogollón, Suárez, Rodríguez, y Fernández, 2016), sobre todo desde el método cualitativo.

Sin embargo, las mujeres continúan esta actividad debido a su rol maternal con el cual están identificadas principalmente. Betancur y Marín (2011) afirman que: "la definición de sí misma está atravesada principalmente por su condición de ser madres" (p. 40). Además este ideal de maternidad, relacionado con el estigma de la prostitución, hace que estas personas mantengan una vida oculta de sus familiares, amigos y de la sociedad en general (Trujillo, 2017).

\section{SUBJETIVIDAD}

Para estudiar a esta población, no se puede limitar sólo a los aspectos cognitivos, también se debe considerar otro aspecto resaltante que es la subjetividad (González, 2009). Pero definir éste término es algo complejo, ya que es un fenómeno dinámico que toma en cuenta la clase, el género, la procedencia, las experiencias vividas, entre otros (Martínez, 2012).

La subjetividad es la representación significante que se produce de la vida social, la cultura y la interrelación con las demás personas (Gallo y Quiñones, 
2016), es decir, no son solo los pensamientos internos, sino que los va construyendo en conjunto con el cúmulo de experiencias, valores, conductas y creencias de la sociedad en la que se desenvuelve, así como también de su historia y cultura.

\section{IDENTIDAD Y SUBJETIVIDAD}

Este proceso se va construyendo de acuerdo al contexto en que se desenvuelve la persona (Bentacur y Marín, 2011), y además posee un componente lingüístico ya que no solo lo utilizamos para definirnos, sino también que se atribuye el discurso que las demás personas realizan de nosotros mismos (Buriticá, 2013). Es por ello que para Revilla (1996) la identidad debe entenderse dentro de la polaridad individuo-sociedad, ya que la considera como un constructo cognitivo que parte del reconocimiento de la percepción que el sujeto posee de sí mismo y a su vez de la intervención de las personas que interactúan con el individuo.

La identidad es un proceso dinámico que está en constante construcción y deconstrucción que se da a lo largo del ciclo vital, y se define en la interacción simbólica con las demás personas (Larrain, 2003). Según Iñiguez (2001), tener plena conciencia de los que nos pasa y de lo que le pasa a los demás es la esencia misma de lo que constituye la identidad. De esta manera, se puede afirmar que la identidad se desarrolla en un entorno social intersubjetivo que viene a ser el centro en donde se constituye la subjetividad, como espacio de interrelación entre lo particular o personal, lo social, lo cultural y lo histórico (Seidmann, 2015).

Sin embargo, lo más destacable en este proceso es lo simbólico y lo emocional de la experiencia humana, la cual se conoce como sentido subjetivo. Un sentido subjetivo es la forma en que un sujeto vive una experiencia subjetivamente, y son las configuraciones subjetivas las que organizan dichas experiencias. Las configuraciones subjetivas son las responsables de motivar el proceso emocional y simbólico sobre la cual se organiza la identidad (González, 2013).

Ahora bien, todas estas teorías vienen fundamentadas desde la investigación de diversos autores, sin embargo ¿cuál es la perspectiva de la mujer en situación de 
prostitución? Es por esta razón que este trabajo tiene como objetivo conocer la identidad y el sentido subjetivo sobre la prostitución en mujeres venezolanas que lo ejercen, por lo que trato de insertarme en este fenómeno desde la mirada de las mujeres en situación de prostitución, teniendo en cuenta que este aspecto es solo una parte de la realidad, ya que la prostitución se manifiesta de diferentes formas.

\section{MÉTODO}

\subsection{Participantes}

El diseño de investigación es cualitativo, el paradigma interpretativo y el método fenomenológico. La selección de las participantes fue intencional y se seleccionaron las que cumplieron con las siguientes condiciones: ser mujeres mayor de edad, venezolana, practicar la prostitución actualmente, ejercer la prostitución en prostíbulos, ser usuaria del Programa de Infecciones de Transmisión Sexual de Barquisimeto (ITS) y que tenga la disposición para participar en la investigación. De esta manera, se seleccionaron 4 mujeres que comprenden una edad entre 24 y 50 años, de nivel socioeconómicos bajos, viven en barrios ubicados al oeste de la ciudad, una posee estudios básicos y las demás diversificados. Todas son madres solteras.

\subsection{Instrumentos}

Para la recolección de información se utilizó la entrevista a profundidad, semiestructurada, por su carácter dialógico que genera un ambiente de confianza para el entrevistador y el entrevistado y de esta manera facilita la comunicación entre ambos (Ozonas y Pérez, 2005). Para la realización de esta entrevista, utilicé como guía el cuestionario realizado por Sánchez y Paz (2005). Además se agregaron otras categorías al cuestionario con la finalidad de complementar la entrevista, el cual contó con la aprobación del Médico Coordinador del área de Epidemiología del ITS, un Msc. en Psicología Social y un Dr. en Psicología que colaboraron con la evaluación del cuestionario final.

\footnotetext{
"No utilizaré la palabra "prostituta" para evitar el estigma social asociado a este término y para no etiquetar a la persona por un solo aspecto de su vida (Cáritas, 2016). También evitaré el término "trabajadora sexual", ya que el mismo designa el ejercicio de la prostitución como una profesión como cualquier otra (Jeffreys, 2011), y además da por entendido que debido a la mujer es que existe la prostitución, ignorando la figura del hombre (Gómez, Pérez y Verdugo, 2016).
} 


\subsection{Procedimiento ${ }^{2}$}

El trabajo de campo se realizó en el año 2017, comenzando con un encuentro privado e individual con cada una de las mujeres que ejercen la prostitución por medio del ITS. Allí mismo, les expliqué sobre la finalidad del trabajo, la confidencialidad, los procedimientos, las técnicas que se utilizarán y si estaban dispuestas a participar. Luego les entregué el consentimiento informado, donde están expresadas las condiciones de la entrevista, las cuales fueron firmadas para dejar constancia de su consentimiento. Seguidamente se realizó la entrevista a profundidad, la cual fue grabada completamente. Una vez terminada, se agradeció a cada participante por su colaboración y se le pidió al representante del ITS que recomendara a otra persona idónea para la realización de la próxima entrevista. Posteriormente se realizó las transcripciones de todas las entrevistas. Al finalizar este procedimiento, realicé el análisis de contenido para conformar las etiquetas de análisis. Al culminar el proceso realicé la categorización de la información recolectada, todo este procedimiento se hizo de forma manual con la aprobación de un Msc. en Psicología Social.

\subsection{Limitaciones}

Poder acceder a estas personas, ya que por la clandestinidad de su oficio, no permiten conversar sobre su realidad. Se intentó realizar una historia de vida y no se logró debido a que las participantes después de 30 minutos manifestaban querer retirarse, además aunque se concretaba una segunda cita no asistían. No todas las personas estaban dispuestas a acceder a la entrevista si no obtenían una gratificación material. La crisis por la que está pasando Venezuela ocasionaba la disminución de asistencia a las instalaciones del ITS, por lo que en ocasiones no se logró realizar ninguna entrevista ya que no asistían a las consultas. Existen pocos datos, cifras oficiales e investigaciones sobre prostitución en Venezuela. La naturaleza misma de los métodos cualitativos los

\footnotetext{
"Los nombres que aparecen en las entrevistas no son sus nombres reales sino sus nombres "artísticos" ya
} que ellas mismas lo sugirieron por ser significativos. 
hace no generalizables, por lo que describen sólo una parte de la realidad. El número de participantes (cuatro mujeres), limita la riqueza de los resultados.

\section{RESULTADOS}

\subsection{Sí misma}

Esta categoría se centra en la perspectiva individual de cada participante acerca de su persona, experiencias de vida y lo que representan a raíz de diversas áreas como la maternidad, relaciones de pareja y percepción de sí misma. Se logró apreciar que son personas con mucha seguridad:

"A nadie le importa si tu comes, si tienes para comprar tus cosas personales, si ayudas a tu familia, si pagas donde vives, si pagas luz, el agua, el cable, la comida, a nadie le interesa eso... tú tienes que estar claro de lo que estás haciendo... y para qué... cuánto tiempo vas a estar ahí, y no te tiene que importar lo que piensen los demás...". (Jennifer).

Esta experiencia ayuda a entender la subjetividad a partir de lo que explica Quiñones (2016), cómo el sujeto se expresa, crea y organiza diferentes experiencias de vida. Todos estos acontecimientos demuestran el cómo se ha forjado la percepción que tienen sobre ellas mismas, es decir, que logran identificarse como personas integrales a partir de las diferentes áreas de su vida como un todo y no sólo por un aspecto como lo es ejercer la prostitución. Esto permite comprender además sus sentimientos de valía a pesar de que no son bien vistas socialmente: “...yo soy una mujer que se valora a sí misma. Si a mí no me valoran yo si me valoro" (María Elena).

Es importante resaltar que un aspecto significativo para ellas son los nombres "artísticos", debido al componente emocional que conlleva, ya que evocan algún recuerdo. Esto va en concordancia con lo explica Páramo (2008) acerca de la identidad, que son:

“las características que posee un individuo, mediante las cuales es conocido... buena parte de la identidad personal la formamos a partir de las interacciones sociales que comienzan con la familia, en la escuela y con la gente que se conoce a lo largo de la vida" (p. 541). 
Esto se puede ver reflejado en la siguiente conversación: “Me llamo Clara. No es mi nombre de pila. Eso me lo pusieron donde trabajé... es que cuando estaba chiquita me decían así, entonces me dijeron: ponte Clara". (Clara).

Este texto expresa una proyección de sí misma que rememora un contexto infantil, por lo que se puede apreciar una interrelación subjetiva que "está constituida tanto en el sujeto individual como en los diferentes espacios sociales en que este vive" (González, 2006, p. 44).

Otro punto importante es la maternidad y la importancia que tiene dentro de su vida cotidiana:

“...los hijos para uno es lo máximo. Yo toda mi vida he estado pendiente de mis muchachos... así yo tenga unos cien años, yo tendré para ellos una arepa con mantequilla y queso o un trago de café... así yo haya hecho lo que haya hecho, esté donde esté, yo siempre estaré ahí para ellos..." (Jennifer).

Betancur y Marín (2011) afirman que: "la definición de sí misma está atravesada principalmente por su condición de ser madres" (p. 40). Desde esta área es su principal motivación para el sostenimiento económico de sus hijos, es por ello que el ser madres es lo que las impulsa a continuar este camino.

Es importante destacar que el área de pareja es el más conflictivo, ya que manejan el imaginario del "hombre de prostíbulo". Este hombre solo busca tener relaciones sexuales y permanecer en una relación a corto plazo sin compromiso personal. Además que el hecho de trabajar en este mundo significa no tener una pareja estable:

“...perjudica que viva con una persona que sepa que yo trabaje ahí... entonces vamos a tener problemas porque yo voy a salir y va a desconfiar. Si me llaman por teléfono voy a tener problemas porque va a estar pendiente, si es celoso puede hasta batí el teléfono...va a estar incómodo...". (María Elena).

Existe también la idea inconsciente de que no merecen ser amadas por una pareja, ya que si visualizan ese mundo como "malo" quiere decir que ellas son 
"malas", por lo que se alejan de cualquier tipo de relación que conlleve un compromiso estable.

En cuanto a la sexualidad, consideran el acto sexual como algo físico y que carece de importancia, mientras que al beso le adjudican un elemento emocional de carácter personal: “...eso muy sublime, es un sentimiento, es algo bien, para venirse a besar con cualquier mamarracho...". (Jennifer).

Es por ello que la corporalidad cumple una función importante en la constitución de la identidad, ya que aunque la mayoría se siente conforme con su cuerpo, la percepción del mismo cambia dependiendo de si están con una persona por dinero o por placer:

“¿Cambia la percepción de su cuerpo cuando está con un cliente? Claro... yo al cliente tengo que coquetiarle... bailale... sobálo... acariciálo... enseñáselas... con mi pareja lo hago pero ya con instinto de placer... con más amor, con más placer y más amistad pues... lo hago con ganas... y con aquellos lo hago es por... por los riales, por Simón Bolívar (el dinero)..." (María Elena).

Según lo anteriormente expuesto, existen dos perspectivas principales en el sentido subjetivo de la identidad. La maternidad, se visualizan como madres y la forma de ganarse la vida responde ante su posición materna. Y la ambivalencia en la estima que tienen de sí mismas al dedicarse a este oficio, aunque manifiestan una autovaloración positiva, también se evidencia el hecho de no permitirse vivir una vida de pareja estable y el cambio en la imagen corporal en relación con los clientes.

\subsection{Narrativas sociales}

Se enfoca en la idea de la sociedad acerca de la prostitución, y sobre los pensamientos de las participantes hacia algunas personas que forman parte de su entorno social.

Según sus relatos, hacen énfasis en un rechazo social hacia su persona: "No, uno no es nada para... para la mayoría de las personas, cuando uno está aquí ¡no vales nada! Por más que tú seas esto, que hables bien, no, no vales nada...” (Jennifer). 
Es por esta razón que existe una ambivalencia en la percepción de la identidad ya que esta surge de la integración de la visión que tienen de sí mismas y la visión que los demás tienen de ellas. Sánchez y Paz (2005), aseguran que surge de un proceso de interacción entre las opiniones de la propia persona y la escucha de la percepción de los demás. En otras palabras, la identidad no está específicamente definida, sino que está constantemente en proceso de construcción de acuerdo a la dinámica personal y social.

Por otro lado, ocultan esta práctica a sus familiares por lo que se limitan a contárselo a personas muy específicas: “...la única que sabe es mi mamá. Que trabajo en esto." (Clara).

Es por ello que tanto la visión de las personas en general y de sus familiares es de vital importancia para el proceso de la identidad, ya que esta se desarrolla en un entorno social intersubjetivo que viene a ser el centro donde se constituye la subjetividad, como espacio de interrelación entre lo personal, social, cultural e histórico (Seidmann, 2015).

La visión que tienen sobre los hombres es que nadie las tomaría en serio debido a cómo se ganan la vida: “¿Qué hombre va a tolerar a una mujer que esté trabajando esto? Que otro poco de hombre la esté agarrando, la esté tocando, la esté penetrando..." (Jennifer).

Acá se puede ver reflejado un auto desprecio de carácter inconsciente hacia lo que se dedican. Es por ello que también hay que prestar atención al lenguaje que utilizan, ya que según Sánchez y Paz (2005): “...la identidad es creada, representada y mantenida a través de las narraciones" (p.15).

Toda esta interrelación de experiencias entre las categorías de sí misma y de narrativas sociales, vienen a revelar la idea central de la construcción subjetiva de la identidad en las participantes, ya que como afirma Iñiguez (2001), tener plena conciencia de los que nos pasa y de lo que le pasa a los demás es la esencia misma de lo que constituye la identidad.

Finalmente, se puede resaltar la ambivalencia en la percepción de la identidad, ya que manifiestan la exclusión y marginación hacia ellas por parte de la sociedad, esconder su labor a familiares y la visión negativa de los hombres hacia ellas. Todo esto las lleva a mantener una perspectiva identitaria confusa, que aunque se reconozcan como seguras de sí mismas y como buenas personas, sus relatos no siempre concuerdan con esta idea. 


\subsection{Narrativas sobre prostitución}

Aquí se aborda la perspectiva de las participantes en donde describen sus vivencias, pensamientos y experiencias con respecto a su día a día y a ellas mismas.

La definición de prostitución fue complicado para expresarlo, ya que conocen lo que es pero se les dificulta enunciarlo: “...lo más bajo, eso no debería existir... no debería oíste..." (Jennifer); "es como un trabajo pero es diferente, es diferente porque... (Se quedó callada)" (Anastasia). Sin embargo dos de ellas coincidieron en el aspecto mercantil de la actividad: “...vender tu cuerpo... (María Elena); "Yo te diría que es vender tu cuerpo" (Clara).

Esto concuerda con el concepto de la Asociación Vivre Ensemble Education (2014), que afirman que el intercambio financiero es lo que estipula este punto del mercado a través de una transacción económica por el cuerpo.

También se puede notar la superioridad numérica de la figura femenina en este mundo con respecto a los hombres, ya que como afirma el OVDHM (2014) quienes solicitan principalmente los servicios de prostitución son los hombres y las personas que la ejercen son mujeres en su mayoría: “ ¿...la prostitución es... principalmente de mujeres? ...por los momento si, es como una base ahorita, para un sostén del hogar". (María Elena).

Por otro lado, es muy latente el imaginario de que el hombre debe establecerse con una mujer "de su casa": "Yo fuera hombre... no voy a tener una relación con una mujer que trabaja en este ambiente". (Jennifer). Es por ello que la subjetividad ayuda a comprenderlo, ya que estudia la representación significante que se produce de la vida social, la cultura y la interrelación con las demás personas (Gallo y Quiñones, 2016). De esta manera se entiende la ambigüedad de que viviendo en una sociedad y cultura machista también adopten este paradigma.

Otro inconveniente es la inseguridad, no solo porque pueden contraer alguna infección de transmisión sexual sino también problemas respiratorios, infecciones en la piel, robos, secuestros, violaciones y un sin número de situaciones: 
“...ahí te pueden puñaliá adentro del baño, un cliente puede matar a uno... alguien que uno le cae mal pueden puñaliá a uno ahí adentro del salón, pueden agarrá a uno a golpes, anda uno corriendo riesgo en la calle... un malandro ve a alguien saliendo de ahí, así tu no haigas trabajao te encapa, te secuestra, te viola, violan a uno y hacen de todo con uno..." (María Elena).

Esto revela los factores de riesgos a los que están sometidas, sin tomar en cuenta la violencia verbal, discriminación y prejuicios por parte de los dueños, compañeras de trabajo, funcionarios públicos, clientes y sociedad en general.

Ante el dilema ¿qué hacer con la prostitución?, ofrecen como opción controlarla ya que aseguran no poder eliminarla: “...yo lo eliminaría... jpor Dios!... pero no se puede, hay que controlarlo..." (María Elena). Sin embargo, según Gálvez (2017) se puede controlar y legalizar este oficio para obtener un amparo judicial pero eso no asegura la aceptación social, por lo que continúa la discriminación, marginación y estigmatización a la cual se ven expuestas diariamente.

Tomando en cuenta lo planteado hasta ahora, se puede decir que el sentido subjetivo que manejan sobre la prostitución es una visión negativa sobre este ambiente, ya que expresan "no haber nada bueno", no tener seguridad, estar expuestas a enfermedades e incluso que debería eliminarse. Sin embargo, hacen la salvedad de que es imposible hacerlo, por lo que debería regularse de alguna manera para que se desarrolle de manera más organizada y que brinde mayor resguardo físico y emocional.

\subsection{Percepción sobre la identidad y la prostitución}

Cada una de sus vivencias en este mundo demuestran la perspectiva subjetiva de cómo ven e interpretan el ambiente en que se encuentran inmersas y el cómo se interpretan a sí mismas. Una de las cosas más llamativas es que la mayoría ingresa por sus propios medios: “¿Quién o quiénes, la ayudaron a involucrarse en este ambiente? No yo misma. Buscando. ¿Por su cuenta? ¡Claro! Averiguando y preguntando llegué a Roma..." (Jennifer). A excepción de Clara: "No... fue mi idea. Fue de mi hermana. La que está allá afuera (señalando)". A diferencia de las demás, este fue el único caso en que un familiar directo la motivó a ingresar ya que también se dedica a este oficio. 
Es importante destacar que Stahl (2015) considera la prostitución como una práctica inherentemente deshumanizante y violenta que no puede ser elegida de forma racional, debido a la presencia de coerción sistémica que es causada por los roles de género desiguales, percepciones desordenadas de la autoestima, y la desventaja económica. Es por ello que esta "libre elección", es el resultado de un contexto socioeconómico que las obliga indirectamente a considerar la prostitución como una forma de obtener un ingreso económico y no elegirla realmente por iniciativa propia.

Así mismo estas bases socio-culturales llevan consigo los pilares fundamentales que complementan el proceso de la identidad, ya que estas mismas personas en otros contextos podrían identificarse con otra labor y dedicarse a otro oficio:

“...yo estaba en Brasil trabajando en un restaurante y... el pasaporte se venció en febrero... el federal me dijo que no podía pasar más porque ya estaba listo... entonces tuve que volver a lo que antes ya había dejado para no estar sin hacer nada... porque no molesto a nadie" (Jennifer).

Las relaciones de amistad no son una opción en este ambiente: Ahí no hay amigos. Ahí hay peligro... maldad, envidia, egoísmo, chisme... buscar aprovecharse de la persona de que por ejemplo te ven chévere y se buscan aprovechar de ti... Para sacarte algún provecho, para que les des algo (Jennifer). Sin embargo, en algunas conversaciones informales con las entrevistadas comentaban que existían ciertas alianzas momentáneas de acuerdo a intereses en común.

Teniendo en cuenta lo planteado, se justifica la permanencia de estas personas en la prostitución debido a los ingresos económicos, que en algunos casos, solo alcanza para cubrir las necesidades básicas:

“...el sueldo mínimo no alcanza y tres hijos más la familia, porque yo mantengo hasta la madre mía, a toda mi familia, a los hermanos y eso y un sueldo mínimo no alcanza para nada y ahora que la comida esta cara". (Anastasia).

Esto demuestra que su identidad está asociada al compromiso maternal, el ser madres es lo que las motiva a continuar en este mundo, sin embargo hay 
semanas en que las ganancias son inferiores al sueldo mínimo según lo expresado por uno de los médicos del ITS.

La integración de los relatos sobre identidad y prostitución, revela algunos aspectos importantes. Como ya se ha mencionado, el factor económico pero principalmente el sentido de la maternidad es lo que las ha impulsado a insertarse en este mundo y sobre todo a mantenerse acá a pesar de los prejuicios, estigmas sociales y personales que esto conlleva. Es por esto que la práctica de la prostitución las hace identificarse de forma ambigua, como madres excepcionales pero no como buenas personas según la sociedad.

\section{CONCLUSIONES}

La prostitución se ha hecho presente en diferentes civilizaciones, lo que ocasiona diversas controversias sobre esta práctica teniendo siempre presente la exclusión social, la marginación, los prejuicios y la estigmatización hacia las mujeres que se dedican a esto (Mogollón et al., 2016), debido a la desigualdad social en que la población femenina se ve mayormente afectada.

Así pues, para dar respuesta al propósito general, la presente investigación ha demostrado que en la identidad se observaron dos perspectivas: la identidad asociado a la maternidad y asociada al auto y hetero rechazo. Esta doble significación se relaciona con lo expuesto por Bajardi (2015), en donde asegura que el proceso de identidad se desarrolla a lo largo de un proceso intersubjetivo que es de naturaleza cambiante, es por ello que varía con el tiempo, la situación y el contexto.

Está asociada a la maternidad ya que están dispuestas a realizar cualquier actividad remunerativa para el sostenimiento de sus hijos. Este ideal de maternidad, que se relaciona con el estigma de la prostitución, hace que estas personas mantengan una vida oculta (Trujillo, 2017), que las hace desarrollar estrategias para mantener esta doble identidad de acuerdo al contexto, esto se ve reflejado en ocultar su conexión con este ambiente a sus familiares y personas en general.

En cuanto al auto y hetero rechazo, manifiestan exclusión y marginación por parte de la sociedad ya que lo consideran como contradictoria al orden moral 
establecido (Gorenstein, 2013). Esto las lleva a mantener una perspectiva identitaria confusa, que aunque se reconozcan como seguras de sí mismas y como buenas personas, sus relatos no siempre concuerdan con esta idea ya que responden a un contexto socio-cultural en la que se encuentran inmersas y por lo tanto adoptan los paradigmas dominantes de la misma. Es así cómo se puede entender el carácter dinámico de la identidad como aseguran Sánchez y Paz (2005) al decir que no existe una sola versión de la identidad establecida por la persona, ya que se reconoce el orden cambiante del mismo.

Es importante destacar que las narrativas sobre la identidad se basaban principalmente en la maternidad y en la ambivalencia de la valía de sí mismas en la moralidad de percibirse como buenas o malas personas. Esto permite comprender la doble realidad en que se encuentran, por un lado identificarse como madres excepcionales, pero a su vez vivir la contradicción de estar realizando algo que no es aprobado socialmente.

Tomando en cuenta lo expresado hasta ahora, puedo decir que la identidad en cada una de las participantes varía de acuerdo al contexto: “...cuando yo estoy en la casa, o con mi familia compartiendo, ¡ni me acuerdo!... que tengo que regresar..." (Jennifer), y aquí es donde se puede apreciar la relación intersubjetiva de la construcción identitaria de cada persona, ya que de acuerdo al contexto y al cómo lo define subjetivamente es el cómo se construyen.

Continuando con el sentido subjetivo de la prostitución, se puede resaltar la visión negativa sobre este ambiente:

“No le desearía esto... Ni a mi peor enemigo. Le diría que trabaje en una zapatería o en una venta de ropa pero aquí no... esto es una pesadilla... tienes que estar... o sea como obligao pues... no, no se lo deseo yo a nadie". (Clara).

Esto está relacionado con la inseguridad, las enfermedades y al manejar el discurso de la abolición de la prostitución. Sin embargo, reconocen que es imposible, por lo que proponen regularla para que se desarrolle de manera más organizada y brinde mayor resguardo físico y emocional. De esta manera se comprende por qué la resistencia a construir un concepto sobre esta práctica, ya que al hacerlo estarían definiendo su estilo de vida. 
Se pudo observar que al hablar de sus realidades lo hacían desde el plano racional, pero al abordar los sentimientos, su lenguaje no verbal cambiaba. Dejaban de mirar a la cara, bajaban sus rostros, unían sus manos... lo que demuestra vergüenza o inseguridad. Esto explica además el hecho de pedir un beneficio material a cambio de realizar la entrevista, ya que se puede continuar con la idea de un intercambio comercial de intereses.

Finalmente en relación a la identidad y la prostitución, es importante mencionar los significados construidos sobre la corporalidad, al cual le otorgan la doble connotación de una sexualidad como forma de trabajo y como expresión de amor. Primero el cuerpo como instrumento de trabajo, sin ningún tipo de conexión emocional: "Una relación más o una relación menos iya para mí es igual!" (Jennifer). La relación sexual en el ambiente de la prostitución carece de goce o disfrute, es por esta razón que aseguran no existir un componente emocional. Sin embargo al ahondar en este tema afirmaban que les genera asco, por lo que negar las emociones durante esta actividad no sería lo correcto.

Y la sexualidad como forma de amar, pude apreciar que existen formas corporales reservadas para la pareja como el beso. Antes de la relación sexual aclaran las normas o describen "el menú" y dicen "sin besos". Es por ello que el sentido subjetivo del cuerpo y de la relación sexual en la prostitución, posee un contenido emocional que es propio de este ambiente.

En resumen, considero tres aspectos esenciales: primero la identidad, se mostraron muy seguras de sí mismas hasta cierto punto, ya que el reconocerse como madres las hace sentirse valiosas por sacar a su familia hacia adelante. Sin embargo, al pensar en ellas desde lo social se describen como marginadas, estigmatizadas y de poco valor, ya que se dedican a una actividad que no es aceptada para la mujer. Es por ello que esta doble realidad les confiere una confusión identitaria desde lo personal y lo social que las llevan a la auto y hetero discriminación.

Segundo, el sentido subjetivo de la prostitución, la describen como algo negativo y mencionan dedicarse a esto por no tener otra opción. Es por ello que lo hacen desde lo racional, esto con la finalidad de no involucrase emocionalmente con esta práctica. 
Sin embargo afirman que la relación sexual debería ser propia de la pareja y no con desconocidos, lo que demuestra su visión negativa no solo para este oficio sino también para ellas mismas. Además que al afirmar que la prostitución debería eliminarse, manifiestan su inconformidad hacia esta práctica. Todos estos aspectos sugieren un carácter emocional, por lo que asegurar que existe una disociación no sería lo correcto.

Por último, puedo decir que existe una ambivalencia en la identidad y el sentido subjetivo sobre la prostitución en las mujeres que lo ejercen, ya que se encuentran atrapadas entre el dilema de la construcción propia sobre sí mismas y la construcción familiar, social, cultural e histórica con que se define el modelo de mujer y el mundo de la prostitución. Es por ello que la interrelación de todas estas estructuras viene a formar una identidad y un sentido subjetivo de la prostitución confusa, ya que responden a un sistema de pensamiento patriarcal propio de nuestra sociedad y que va en discordancia con el ideal de mujer y de madre.

Además, se recomienda para futuras investigaciones continuar con las investigaciones sobre identidad, subjetividad y prostitución en el área de la Psicología, sobre todo en el contexto venezolano, que contribuyan a comprender más esta realidad y a la creación de políticas públicas e intervenciones psicosociales que permitan abordar tanto este ambiente como a estas personas. Se considera oportuno definir un tiempo determinado en el ejercicio de la prostitución para la elección de las participantes, ya que se observó que mientras más años tienen en esta práctica mejor es la calidad de la información. Es recomendable también ampliar el número de participantes para una mayor riqueza de la información.

\section{BIBLIOGRAFÍA}

Agrela, B., Martín Palomo, M. T., Langa, D. (2010). Modelos de provisión de cuidados: género, familias $\mathrm{y}$ migraciones. Nuevos retos $\mathrm{y}$ configuraciones. Alternativas. Cuadernos de Trabajo Social, 17, 9-17. Doi: https:/ / doi.org/10.14198/ ALTERN2010.17.1

Álvarez, A. (2012). La prostitución de mujeres, una escuela de desigualdad humana. Revista Europea de Derechos Fundamentales, 19, 49-74. 
Aragón, J. (2000). Variables cognitivo-conductuales y VIH en trabajadoras sexuales del eje La Fría - San Antonio Rubio (Estado Táchira) en la frontera de Venezuela con Colombia. Acción Pedagógica, 9, 58-67.

Asociación Vivre Ensemble Educatión. (2014). La prostitution: un choix? (1er. ed.). Francia.

Bajardi, A. (2015). La identidad personal en relación con la educación: características y formación del concepto. REIDOCREA, 15, 106-114. doi: https: / / doi.org/10.30827/Digibug.37124

Betancur, C., y Marín, A. (2011). Cuerpo, comercio sexual, amor e identidad. Significados construidos por mujeres que practicaron la prostitución. Revista CES Psicología, 4(2), 32-51.

Buriticá, I. (2013). Travesti: la construcción de la identidad individual y colectiva desde el cuerpo y el ejercicio de la prostitución. La Manzana de la Discordia, 8(2), 71-86. doi: https:/ / doi.org/10.25100/Imd.v8i2.1541

Cáritas, (2016). La prostitución desde la experiencia y mirada de Cáritas. (1ra. ed.). Madrid, España. Cáritas Española Editores.

Condiza, W., y Hernández, E. (2012). Pobreza y prostitución en Boyacá, Colombia: una mirada desde los derechos humanos. Revista Colombiana de Sociología, 35(1), 83-95.

Dakduk, S. (2007). El trabajo sexual femenino: el caso venezolano en el 2006. Revista Venezolana de Estudios de la Mujer, 12(29), 135-148.

De Matos, M., Amorim, K., Dias, D., Silva, R., Carneiro, L., y Araujo, S. (2013). La vulnerabilidad a las enfermedades de transmisión sexual en mujeres que venden sexo en ruta de prostitución y turismo sexual en Brasil Central. Rev. Latino-Am. Enfermagem, 21(4), 01-07. doi: https: / / doi.org/10.1590/S0104-11692013000400011

Díez, E. (2012). El papel del hombre en la prostitución. Nuestra Bandera, 232, 39-54.

Fernández, M. (2011). Calidad de vida y salud en mujeres que ejercen la prostitución. (Tesis Doctoral, Universidad de Oviedo, España). Recuperada de: http:/ / dspace.sheol.uniovi.es/dspace/ bitstream/10651/12712/1/TD_ MariaJesusFernandezOllero.pdf

Fundation Scelles. (2012). L'Exploitation de la prostitution: un fléau mundial. (3ra. ed.). Pulnoy, Francia.

Gallo, J., y Quiñones, A. (2016). Subjetividad, salud mental y neoliberalismo en las políticas públicas de salud en Colombia. Athenea Digital, 16(2), 139168. doi: http: / / dx.doi.org/10.5565/ rev/athenea.1616

Gálvez, A. (2017). La prostitución reglamentada en latinoamérica en la época de la modernización. Los casos de Argentina, Uruguay y Chile entre 1874 y 1936. Historia 396, 1, 89-118. 
Gómez, A., Pérez, S., y Verdugo, R. (2016). Dominación, sexualidad masculina y prostitución en España: ¿por qué los hombres españoles consumen sexo de pago? Convergencia Revista de Ciencias Sociales, 71, 149-174. doi: https:/ / doi.org/10.29101/ crcs.v0i71.3993

Gómez, A., y Verdugo, R. (2015). La prostitución femenina en España. Construyendo un perfil del cliente. Papeles de Población, 21(86), 9-39.

González, F. (2006). Investigación cualitativa y subjetividad. Guatemala. ODHAG.

González, F. (2009). Epistemología y Ontología: un debate necesario para la Psicología hoy. Revista Diversitas - Perspectivas en Psicología, 5(2), 205224. doi: https:/ / doi.org/10.15332/s1794-9998.2009.0002.01

González, F. (2013). La subjetividad en una perspectiva cultural-histórica: avanzando sobre un legado inconcluso. $C S, 11,19-42$. doi: https:/ / doi.org/10.18046/ recs.i11.1565.

Gorenstein, S. (2013). Prostitución: permitida y estigmatizada. Perspectivas discursivas a partir de las historias de vida de seis mujeres que se prostituyen en El Trocadero. Debates en Sociología, 38, 29-54.

Iñiguez, L. (2001). Identidad: de lo personal a lo social. Un recorrido conceptual. En Eduardo Crespo (Ed.), La constitución social de la subjetividad. (p. 209-225). Madrid: Catarata.

Jeffreys, S. (2011). La industria de la vagina. La economía política de la comercialización global del sexo. Buenos Aires, Argentina. Paidós.

Larrain, J. (2003). El concepto de identidad. Revista FAMECOS, 21, 30-42. Doi: https:/ / doi.org/10.15448/1980-3729.2003.21.3211

Martínez, S. (2012). Ser o no ser: tensión entre familia, subjetividad femenina y trabajo académico en Chile. Un análisis desde la psicología feminista. Revista de Estudios de Género La Ventana, 35(4), 133-163.

Mogollón, E., Suárez, F., Rodríguez, M., y Fernández, H. (2016). El estigma de la prostitución y los derechos humanos. Revista Venezolana de Estudios de la Mujer, 21(47), 102-113.

Observatorio Venezolano de los Derechos Humanos de las Mujeres [OVDHM]. (2014). Recuperado de: http:/ / observatorioddhhmujeres.org/documentos / informe\%20alternat ivo.htm\#VI.

Ozonas, L., y Pérez, A. (2005). La entrevista semiestructurada. Notas sobre una práctica metodológica desde una perspectiva de género. La Aljaba, 9, 198-203.

Páramo, P. (2008). La construcción psicosocial de la identidad y del self. Revista Latinoamericana de Psicología, 40(3), 539-550.

Quiñones, G. (2016). 'Visual Vivencias' to understand subjectivity and affective connection in young children. Video Journal of Education and Pedagogy, 1(3), 1-14. doi: https: / / doi.org/10.1186/s40990-016-0004-1 
Revilla, J. (1996). La identidad personal en la pluralidad de sus relatos. Estudio sobre jóvenes. (Tesis Doctoral, Universidad Complutense de Madrid, España). Recuperada http:/ / biblioteca.ucm.es/tesis / 19911996/S/1/S1018201.pdf

Sánchez, A., y Paz, C. (2005). Construcción de identidad en mujeres adultas que ejercen la prostitución vinculadas a un programa de ayuda estatal. (Trabajo de Grado, Pontificia Universidad Javeriana, Bogotá, Colombia). Recuperada http:/ / javeriana.edu.co/biblos/tesis/psicologia/tesis20.pdf

Seidmann, S. (2015). Identidad personal y subjetividad social: educación y constitución subjetiva. Cuadernos de Pesquisa, 45(156), 344-357. doi: http:/ / dx.doi.org/10.1590/198053143204

Stahl, K. (2015). Addressing Demand for Sex Trafficking in Sweden and the United Kingdom: An Interpretive Policy Analysis of Demand Reduction Policies, in Consideration of the Principles of Deterrence Theory. (Trabajo de Grado de Maestría, Reino Unido). Recuperado de: http: / / scholarworks.gvsu.edu/cgi/viewcontent.cgi?article=1753\&cont ext=theses

Trujillo, M. (2017). Maternidad y prostitución ¿contradictorias y excluyentes?. Revista Estudios Feministas, 25(1), 163-181. doi: https: / / doi.org/10.1590/1806-9584.2017v25n1p167

Ulloa, T. (2011). La prostitución: una de las expresiones más arcaicas y violentas del patriarcado contra las mujeres. Pensamiento Iberoamericano, 9, 293-312. 